\title{
Family-Oriented Social Service Touchpoints as Opportunities to Enhance Diabetes Screening following a History of Gestational Diabetes
}

\author{
Maya Venkataramani, MD, MPH, Tina L. Cheng, MD, MPH, Hsien-Chieh Yeh, PhD, \\ Wendy L. Bennett, MD, MPH, and Nisa M. Maruthur, MD, MHS
}

Introduction: Women with a history of gestational diabetes (GDM) are at increased risk for type 2 diabetes and thus require regular follow-up screening for diabetes; however, many women do not receive this screening, and in particular low-income women face disparities in receipt of recommended follow-up care. While these women may have limited access to healthcare following pregnancy, they may more regularly access social service programs that serve themselves or their young children. Leveraging these social service touchpoints could broaden opportunities to improve follow-up care receipt among women with a history of GDM. To describe these potential opportunities, we used national representative data to characterize diabetes screening needs among women with a history of GDM who access the Special supplemental nutrition program for Women, Infants and Children (WIC) or Head Start programming for their young children.

Methods: We analyzed national representative data from the National Health Interview Survey from calendar years 2016 and 2017. Our analytic sample included women aged 18 to 45 years who were linked to at least one of their children in the dataset and who had a self-reported history of GDM but did not have prediabetes or diabetes. We examined the proportion of these women who accessed WIC or Head Start who did not report having testing for diabetes within the past 3 years.

Results: of 432 (representing 2,002,675 weighted) women meeting inclusion criteria, $21.7 \%$ accessed WIC and $8.7 \%$ Head Start. Nearly 1 in 10 women with a history of GDM in either group did not report recent diabetes screening. In sensitivity analyses that excluded likely pregnancy-related testing, 35.0\% of women accessing WIC and $21.2 \%$ of those accessing Head Start had not had recent screening.

Discussion: There is an unmet need for follow-up diabetes screening among women with a history of GDM who access WIC or Head Start services for their young children. Leveraging women's touchpoints with these programs could enhance opportunities to improve recommended diabetes screening among a high-risk population. (J Am Board Fam Med 2020;33:616-619.)

Keywords: Follow-Up Care, Gestational Diabetes, Health Care Disparities, Prediabetic State, Pregnancy, Risk Factors, Screening, Social Work, Type 2 Diabetes, Women's Health

\section{Introduction}

Women with a history of gestational diabetes $(\mathrm{GDM})$ are at increased risk for type 2 diabetes. As such, guidelines recommend follow-up diabetes screening among women with GDM at 4 to

This article was externally peer reviewed.

Submitted 21 October 2019; revised 3 February 2020; accepted 6 February 2020.

From the Division of General Internal Medicine, Johns Hopkins University School of Medicine, Baltimore, MD (MV, HCY, WLB, NMM); Department of Pediatrics, Johns Hopkins University School of Medicine, Baltimore, MD (MV, TLC, NMM); Welch Center for Prevention, Epidemiology and Clinical Research, Baltimore, MD (MV, HCY, WB, NMM); Johns Hopkins Brancati Center for the Advancement of Community Care, Baltimore, MD (MV, NMA).
12 weeks postpartum and at least every 1 to 3 years thereafter. ${ }^{1,2}$ Unfortunately, few women with a history of GDM in the United States receive recommended follow-up diabetes screening, representing a significant missed opportunity for early detection or initiation of prevention activities. Studies reveal the

Funding: Dr. Venkataramani was supported by the Johns Hopkins Clinician Scientist Award program and is supported by the NIDDK of the National Institutes of Health under award number K23-DK119781.

Conflicts of interest: No competing interests.

Corresponding author: Maya Venkataramani, MD, MPH, Johns Hopkins University School of Medicine Division of General Internal Medicine 2024 E Monument Street, 2-502 Baltimore, MD 21287 (E-mail: mvenkat2@jhmi.edu). 
percentage of women with a history of GDM who receive diabetes screening in the 3 months to 3 years following delivery ranges from $5.7 \%$ to $57.9 \%$, and that screening prevalence is particularly poor among low-income women. ${ }^{3-5}$ Further, it has been found that certain racial and ethnic minorities and women with public insurance coverage are less likely to be screened. ${ }^{3}$ Multiple reasons have been cited for poor followup, including limited interactions with the health care system postdelivery period. ${ }^{3,5}$

In this crucial period, lower-income women may have more regular contact with social service providers, particularly organizations that serve their young children such as the early education service, Head Start, or the Special Supplemental Nutrition Program for Women, Infants, and Children (WIC), both which serve low-income children up to the age of 5 years. WIC also provides services to women during pregnancy and in the postpartum period. Even if the parent is not receiving services directly, there is precedence for these programs to address health-related issues of parents and caregivers. ${ }^{6,7}$ Leveraging these social service touchpoints could thus broaden opportunities to improve follow-up care among women with a history of GDM, through a range of possible interventions from enhances awareness regarding the need for diabetes screening to linking women to primary care services to potentially performing screening at these sites. To describe these potential opportunities, we used nationally representative data to characterize diabetes screening needs among women with a history of GDM who access WIC and Head Start.

\section{Methods}

We analyzed data from the 2016 and 2017 National Health Interview Survey (NHIS), obtained from the Integrated Public Use Microdata Series database. ${ }^{8}$ The NHIS is an annual survey of a nationally representative sample of US households. The survey collects information on a range of topics among household members, including health status, and health and social service utilization. Our analytic sample included women ages 18 through 45 years who were not currently pregnant and had a self-reported history of GDM (in any prior pregnancy). We further restricted the sample to women who could be linked to at least 1 child (ages 17 years or younger) who was also represented in the dataset. We excluded women with self-reported prediabetes or diabetes.
The NHIS collects information about selfreported utilization of WIC among women and children and Head Start enrollment among children. Our primary analyses focused on 1) women who had received WIC services for themselves or any of their children in the past calendar year, and 2) women with a child currently enrolled in Head Start. Our outcome was self-reported diabetes screening in the past 3 years.

We performed descriptive analyses to characterize relevant demographic characteristics available in the NHIS data, including race, ethnicity, income, insurance coverage and whether the woman had a visit with a medical provider in the past year. We calculated the proportion accessing WIC and Head Start who had not received recent screening. All analyses took into account the NHIS's complex survey design and associated survey weights.

As the self-reported diabetes screening measure may have been interpreted by respondents to include diabetes-related testing occurring during pregnancy (that is, testing related to GDM screening during pregnancy), we conducted a sensitivity analysis that would exclude likely pregnancy-related testing (eg, only counting screening occurring within the past 2 years if the youngest child was 2 years old).

\section{Results}

Of 432 (representing 2002,675 weighted) women meeting inclusion criteria, $21.7 \%$ accessed WIC and $8.7 \%$ Head Start. Women accessing these services were predominantly low income, with high prevalence of uninsured status (Table 1). We found that $10.5 \%$ (95\% CI, $5.0 \%$ to $20.7 \%)$ of women with a history of GDM accessing WIC services and $8.2 \%$ of women with a history of GDM accessing Head Start services (95\% CI, $2.9 \%$ to $21.0 \%$ ) did not report recent diabetes screening (Table 2). In sensitivity analyses (excluding likely pregnancyrelated testing), $35.0 \%$ of women with a history of GDM accessing WIC and $21.2 \%$ of those accessing Head Start did not report recent screening.

\section{Discussion}

Among women with a history of GDM who access WIC or Head Start, nearly 1 in 10 have not had recent diabetes screening, and this proportion may actually be 3-fold higher (as the self-reported screening measure may have actually reflected testing for 
Table 1. Selected Characteristics of Women with a History of Gestational Diabetes* from the National Health Interview Survey (2016 and 2017), by Special Supplemental Nutrition Program for Women, Infants, and Children (WIC) or Head Start Access Status

\begin{tabular}{|c|c|c|c|}
\hline & $\begin{array}{c}\text { Women Accessing WIC } \\
\text { Services }{ }^{\dagger} \\
\% \text { of } \mathrm{N}^{\S}(95 \% \mathrm{CI})\end{array}$ & $\begin{array}{c}\text { Women Accessing Head } \\
\text { Start Services }{ }^{\dagger} \\
\% \text { of } N^{11}(95 \% \text { CI })\end{array}$ & $\begin{array}{c}\text { Women Accessing Neither } \\
\text { Service } \\
\% \text { of } N^{\dagger}(95 \% \text { CI })\end{array}$ \\
\hline Age, years (mean, (SE)) & $31.8(0.91)$ & $34.5(1.24)$ & $36.8(0.42)$ \\
\hline \multicolumn{4}{|l|}{ Race } \\
\hline White & $77.9(65.3$ to 86.9$)$ & $66.1(41.2$ to 84.4$)$ & $73.9(67.2$ to 79.6$)$ \\
\hline African-American & $8.0(4.1$ to 15.0$)$ & $27.1(10.8$ to 53.3$)$ & $10.6(7.1$ to 15.7$)$ \\
\hline $\begin{array}{l}\text { Asian, Alaskan Native/ } \\
\text { American Indian, other }{ }^{\pi}\end{array}$ & $14.1(6.4$ to 28.1$)$ & $6.8(1.7$ to 23.9$)$ & 15.5 (10.5 to 22.2) \\
\hline Hispanic Ethnicity ${ }^{\#, * *}$ & $38.3(25.8$ to 52.6$)$ & $39.1(18.3$ to 65.0$)$ & 15.8 (11.6 to 21.2) \\
\hline \multicolumn{4}{|l|}{ Income category ${ }^{\#, * *}$} \\
\hline$<100 \% \mathrm{FPL}$ & $38.9(26.2$ to 53.3$)$ & $37.4(18.4$ to 61.3$)$ & 9.5 (6.6 to 13.5$)$ \\
\hline $100 \%$ to $<200 \% \mathrm{FPL}$ & $42.2(27.9$ to 57.9$)$ & $26.8(8.7$ to 58.4$)$ & 17.1 (12.6 to 22.8) \\
\hline$\geq 200 \% \mathrm{FPL}$ & $18.9(10.2$ to 32.3$)$ & $35.8(18.3$ to 58.1$)$ & 73.4 (67.8 to 78.3$)$ \\
\hline \multicolumn{4}{|l|}{ Current insurance coverage ${ }^{\#, * *}$} \\
\hline Uninsured & $15.7(8.2$ to 28.0$)$ & $5.2(1.4$ to 17.4$)$ & $8.2(5.2$ to 12.7$)$ \\
\hline Public & $45.6(31.9$ to 60.1$)$ & $41.0(20.7$ to 64.9$)$ & $14.0(9.6$ to 20.0$)$ \\
\hline Any private & $38.7(26.8$ to 52.1$)$ & $53.8(30.7$ to 75.4$)$ & $77.8(71.2$ to 83.3$)$ \\
\hline $\begin{array}{l}\text { Had a visit with a medical } \\
\text { provider in the past } 1 \text { year }{ }^{\#}\end{array}$ & $85.2(69.6$ to 93.5$)$ & $57.2(31.8$ to 79.4$)$ & $86.2(80.2$ to 90.6$)$ \\
\hline
\end{tabular}

FPL, federal poverty level; SE, standard error; CI, confidence interval; NHIS, national health interview survey. All descriptive statistics calculated using NHIS sample weights.

${ }^{*}$ Women ages 18 to 45 years without self-reported diabetes or pre-diabetes with a child linked in the dataset.

${ }^{\dagger}$ WIC services for self or child in prior calendar year; child currently enrolled in Head Start.

${ }^{\ddagger}$ Women who may or may not have had a child in the dataset.

In =1,438,683 weighted (316 unweighted).

$\$_{\mathrm{n}}=434,540$ weighted ( 87 unweighted); for income, race, visit in past year, $\mathrm{n}=422,228$ weighted ( 86 unweighted).

" $\mathrm{n}=174,104$ weighted (37 unweighted); for race and Hispanic ethnicity, $\mathrm{n}=173,025$ weighted (36 unweighted).

"More than 1 race.

\#Indicates categories with significantly different distribution among women accessing WIC compared to women accessing neither service (by Pearson's $\chi^{2}$ test).

**Indicates categories with significantly different distribution among women accessing Head Start compared to women accessing neither service.

GDM during pregnancy rather than diabetes screening after pregnancy for some women). Overall, nearly one quarter of women ages 18 through 45 years with a history of GDM who have not received recent diabetes screening could be reached by leveraging their WIC or Head Start touchpoints. In particular, these programs provide access to a population with several risk factors for poor health care access, such as poverty and lack of insurance, in the postpartum period and beyond.

Interventions to enhance follow-up care could include awareness campaigns and providing linkages to insurance and primary care. There is precedence for WIC and Head Start programs to provide such linkage to care services to parents and caregivers. ${ }^{6,7}$ If there exists an ability to link to care (and thereby ensure follow-up of abnormal screening tests), offering initial screening for type 2 diabetes could prove particularly effective. Hemoglobin A1c or fasting glucose can be performed by point-of-care testing and are considered appropriate screening tests after 12 weeks postpartum. This screening may be particularly feasible at WIC sites, which already perform certain point-of-care blood testing for children and pregnant and postpartum women accessing their services. Having a system through which screening results could be communicated easily with primary care providers would be important in ensuring appropriate follow-up care occurs. Systematically addressing GDM followup at these sites could also include the provision of lifestyle programming designed to prevent diabetes among these high-risk women. ${ }^{9}$

It is also worth noting that a high proportion of women in our sample reported seeing a medical 
Table 2. Prevalence of Recent Diabetes Screening among Women with a History of Gestational Diabetes* from the National Health Interview Survey (2016 and 2017), by Special Supplemental Nutrition Program for Women, Infants, and Children (WIC) or Head Start Access Status

\begin{tabular}{lcc}
\hline & $\begin{array}{c}\text { Women Accessing WIC Services } \\
\% \text { of N }(95 \% \text { CI })\end{array}$ & $\begin{array}{c}\text { Women Accessing Head Start Services }^{\dagger} \\
\% \text { of N (95\% CI) }\end{array}$ \\
\hline $\begin{array}{l}\text { Has NOT had diabetes screening within } \\
\text { the past 3 years }\end{array}$ & $10.5(5.0$ to 20.7) & $8.2(2.9 \text { to 21.0 })^{\S}$ \\
$\begin{array}{l}\text { Sensitivity analysis: Has NOT had diabetes } \\
\text { screening within past 3 years } \text { or after most } \\
\text { recent pregnancy }\end{array}$ & $35.0(21.8$ to 51.0)\# & $21.2(9.3 \text { to } 41.3)^{* *}$ \\
\hline
\end{tabular}

All descriptive statistics calculated using NHIS sample weights.

${ }^{*}$ Women ages 18 to 45 years without self-reported diabetes or pre-diabetes with a child linked in the dataset.

${ }^{\dagger}$ WIC services for self or child in prior calendar year; child currently enrolled in Head Start.

"Self report of receipt of "last blood test for high blood sugar/diabetes."

$\$_{\mathrm{n}}=422,228$ weighted (86 unweighted).

In $=174,104$ weighted (37 unweighted).

${ }^{\pi}$ Self-reported screening in past 3 years OR if had a child 2 years or younger, occurring after recent pregnancy; $\mathrm{n}=327,874$ weighted (64 unweighted).

${ }^{* *} \mathrm{n}=147,574$ weighted (32 unweighted).

NHIS, national health interview survey; CI, confidence interval.

provider in the past year. While this may not necessarily reflect a primary care provider encounter, studies have shown that there are missed opportunities for diabetes screening among those women who do access primary care. ${ }^{5}$ Understanding what patient- or provider-related factors impact screening receipt during primary care encounters would also be important to informing an overarching approach to improve diabetes screening among women with a history of GDM.

Limitations of this work include the use of a selfreported screening measure that does not distinguish postpartum diabetes screening from pregnancy-related GDM screening. In addition, women may be unaware of being screened or incorrectly recall the screening period. Further, estimates were based on small sample sizes. Future work should confirm the prevalence of overdue diabetes screening among women with a history of GDM who access WIC and Head Start, characterize reasons for missed follow-up, and explore the feasibility of delivering targeted interventions at program sites.

To see this article online, please go to: http://jabfm.org/content/ 33/4/616.full.

\section{References}

1. American Diabetes Association. Management of diabetes in pregnancy: standards of medical care in diabetes-2019. Diabetes Care 2019;42:S165-S172.

2. American College of Obstetrics and Gynecology. Practice Bulletin No. 190. Gestational Diabetes Mellitus. Obstet Gynecol 2018;131:e49-64.
3. Jones EJ, Hernandez TL, Edmonds JK, Ferranti EP. Continued disparities in postpartum follow-up and screening among women with gestational diabetes and hypertensive disorders of pregnancy: a systematic review. J Perinat Neonatal Nurs 2019;33:136-48.

4. Bennett WL, Chang HY, Levine DM, et al. Utilization of primary and obstetric care after medically complicated pregnancies: an analysis of medical claims data. J Gen Intern Med 2014;29:636-45.

5. Ferrera A, Peng T, Kim C. Trends in postpartum diabetes screening and subsequent diabetes and impaired fasting glucose among women with histories of gestational diabetes mellitus. Diabetes Care 2009;32:269-74.

6. Ibrahim A. CCI links WIC participants to primary \& preventative services in Montgomery County, MD. Available from: https://www.acog.org/-/media/ Departments/Public-Health-and-Social-Issues/ Community-Clinical-Linkages-Success-Story-1. pdf?dmc $=1 \&$ ts $=20190628 \mathrm{~T} 2116442691$. Accessed June 28, 2019.

7. Aikens N, Tarullo L, Hulsey L, Ross C, West J, Xue Y. A year in Head Start: children, families, and programs. Washington, DC: U.S. Department of Health and Human Services, Administration for Children and Families, Office of Planning, Research, and Evaluation; 2010.

8. Blewett L, Rivera D, Griffin R, King M, Williams CW. IPUMS Health Surveys: National Health Interview Survey, Version 6.3 [dataset]. Minneapolis, MN: IPUMS, 2018.

9. Rosal MC, Lemon SC, Nguyen OHT, et al. Translation of the diabetes prevention program lifestyle intervention for promoting postpartum weight loss among low-income women. Behav Med Pract Policy Res 2011;1:530-8. 